\title{
The evolution of children hearing loss: Research progress on recent trends and advances
}

\author{
Xinyang Wang ${ }^{1, a}$ \\ ${ }^{1}$ Department of Rehabilitation Sciences, Hong kong Polytechnic University, Hong kong,999077, China
}

\begin{abstract}
Hearing impairment is a common disease, affecting about five percent of the population. It puts great pressure on children, their families and society, and has become an significant problem affecting daily life. In recent years, great progress has been made in the study of hearing loss in children. This paper describes the ear structure, the causes of hearing impairment, the assessment and diagnosis of hearing impairment and the current applicable treatment methods through literature review. Previous research on hearing loss in children is summarized in this paper and provides some references for the further research.
\end{abstract}

\section{INTRODUCTION}

More than five percent of the world's population has some degree of hearing impairment [1]. Hearing loss is classified as mild (26-40 dB HL), moderate (61-80 dB $\mathrm{HL})$ or profound $(81 \mathrm{~dB} \mathrm{HL}$ or greater). For children, hearing level of $35 \mathrm{~dB} \mathrm{HL}$ is considered as disabled. Hearing impairment is a great burden to children, family and society, and has become one of the important issues affecting personal life. Therefore, it is important to carry out research on hearing impairment. There is ample evidence that early intervention is a key factor in achieving optimal treatment outcomes [2]. Under the same condition of cochlear implantation, patients at a younger age of implantation, use a hearing aid before surgery and with cochlear morphology, will have better audiological and speech outcomes [3].

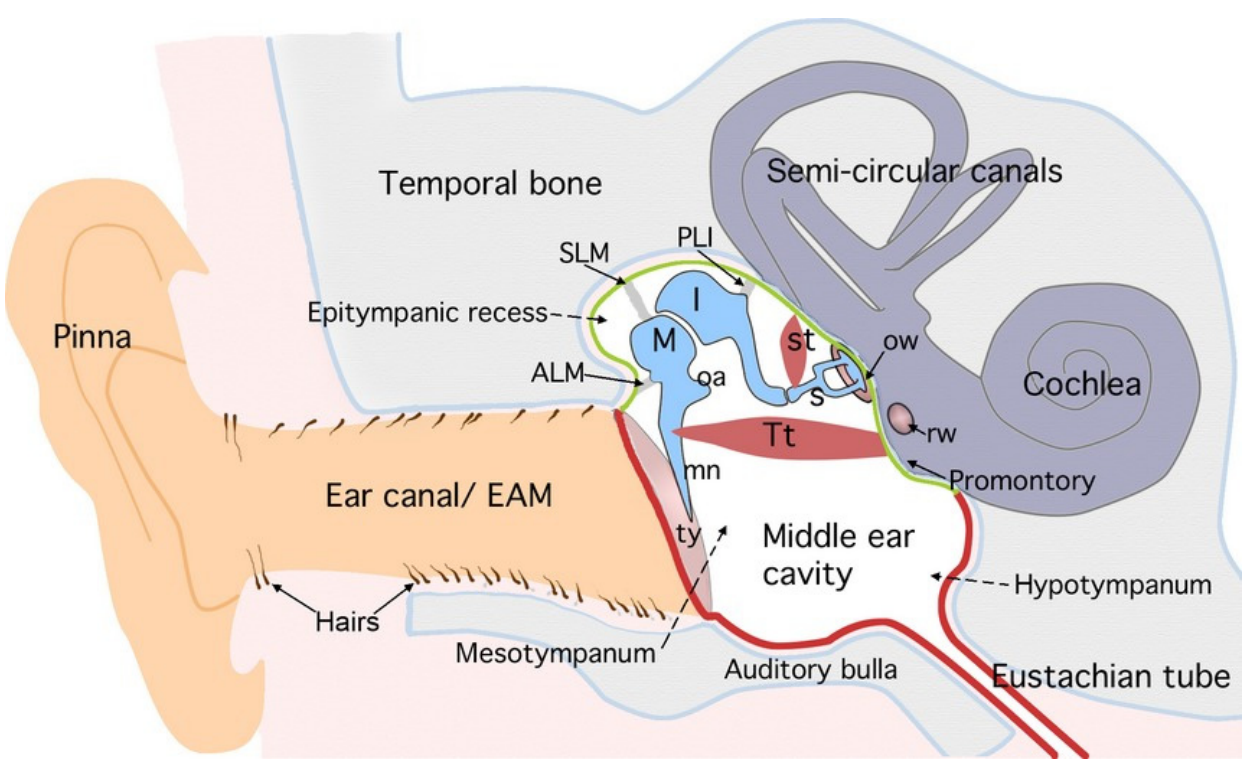

Figure 1. Anatomy of the ear[4]

The ear can be divided into three structures, the external, the middle and the inner [5], and the specific ear structure is shown in figure 1. The external ear is composed of the pinna and the ear canal. Its function is to direct sound waves from the ear canal to the eardrum. The middle ear is an air-filled cavity in the temporal bone of the skull. The ossicular bone in the middle ear vibrates sound waves and sends them into the inner ear. Inside the cochlea, in the inner ear, sound waves are converted into electrochemical signals that are transmitted to the brain. The inner ear is involved in hearing and balance[4].Hearing loss can be divided into 
three categories, namely sensorineural hearing loss, conductive hearing loss and mixed hearing loss. Developmental defects in the external and middle ears can lead to conductive hearing loss[4].Cerumen impactive, otitis media and otosclerosis can lead to conductive hearing loss. Otitis media is one of the most common causes of conductive hearing loss in children. As for sensorineural hearing loss, in pediatrics there are two main types of triggers: hereditary and non-hereditary. [6]. Besides, developmental defects in the inner ear can lead to sensorineural hearing loss [4]. Most patients with this type of infection are adults. Among non-genetic causes, noise exposure is the most common preventable factor [7].

At present, the assessment methods are mainly objectively measured by audiologists,including pure-tone average, speech recognition thresholds and word recognition scores, while subjective assessment is mainly achieved by validated questionnaire survey among parents and teachers, such as The Parents' Evaluation of Aural/Oral Performance of Children and Teachers' Evaluation of Aural/Oral Performance of Children[8].

Cochlear implantation is an established procedure for the treatment of severe hearing impairment[3]. In the treatment of unilateral hearing loss, non-surgical methods, like FM system (a conventional choice for patients with mild to profound UHL) has a good effect for patients with mild to moderate UHL, which is more effective than conventional hearing aids. And bone-conduction hearing devices implanted surgically have significant therapeutic effects on moderate to profound UHL patients [8]. According to recent papers, there are new technologies in children's hearing therapy including the group of family involvement(GrAF) [9] and microprocessors, computer-based software training and tele-health and so on[10].

In this paper, the causes of hearing impairment were concluded through literature analysis of articles in the past 10 years, and the commonly used assessment methods and treatment schemes were summarized. Treatment options will be described from the perspective of different populations. Meanwhile, parents and software adjuvant therapy will also be mentioned in this paper. The purpose of this paper is to describe the objective functional effects of current hearing rehabilitation programs through literature review, and to provide some references for future research on children hearing impairment.

\section{The causes of hearing loss}

As for the causes of hearing loss, genetic causes account for 40 percent of hearing loss in children[7]. The underlie for hearing impairment in most people seems to be a complete loss of ILDR1 function. Mutations in the ILDR1 gene constitute a cause of DFNB hearing impairment. [11]. Lysyl-tRNA synthetase (KARS) plays an important role in the localization of cochlea, and the mutation of this gene could be the cause of the hearing impairment that infects autosomal-recessive nonsyndromic hearing impairment (ARNSHI ) [12]. Stereocilin(STRC) is a major cause of congenital hearing impairment, caused by mutations in this gene contribute to non-syndromic sensorineural hearing loss (NSHL), which is particularly suitable for children with mild to moderate hearing impairment and has a greater impact and higher frequency[13].

On the other hand, there are three main causes of acquired hearing loss: external ear, middle ear, and inner ear impairment. First of all, as for external ear, complete occlusion of the ear canal is a common cause of unilateral conductive hearing loss, which can be removed with an otoscope and a curette. Similarly, there are foreign bodies in the external auditory canal, which can be removed by irrigation or with a curette. Otitis externa can also lead to conductive hearing loss if the patient's canal is swollen and filled with debris[7].The most common reason are Pseudomonas aeruginosa and Staphylococcus aureus infection[14].

Secondly, when it comes to middle ear, Otitis media is the most common cause of temporary and conductive hearing loss in children[15, 16], including acute otitis media, tympanum membrane perforation, and otitis media with effusion or glue ear. Infections in children are the cause of otitis media. Children with a history of acute otitis media or recurrent otitis media have a higher risk of developing chronic otitis media and recurrent otitis media in the future[17]. Otitis media can be determined by tympanogram and audiometry[7].

Thirdly, in terms of inner ear, noise exposure is the most common preventable cause of non-genetic [7]. Excessive exposure can lead to noise-induced hearing loss, which can be occupational, recreational or sporadic. Hearing loss caused by recreational noise is more likely to occur in adolescents[17]. The use of noise protection can effectively prevent this type of sensorineural hearing loss[7]. In addition, the use of ototoxic drugs in neonates also leads to hearing loss in the inner ear, accounting for 4 percent of the hearing loss causes[17]. Common drugs include aminoglycosides and cisplatin, used to treat tuberculosis and cancer, respectively[18, 19], which can lead to permanent hearing loss[20].

\section{Auditory Assessment Methods}

Some commonly used methods for assessing hearing impairment are The Rinne test and Weber test, Pure tone audiometry, sound-steady State Response and The Parents'/Teachers' Evaluation of Aural/Oral performance of Children(PEACH/TEACH).

\subsection{The Rinne test and Weber test}

The Rinne test is to determine whether the patient has conductive hearing loss or sensorineural hearing loss by comparing the bone conduction and air conduction of the patient. The test involves placing a vibrating tuning fork in the patient's ear near the mastoid bone to measure bone conduction. When the patient is unable to hear, the tuning fork is placed near the ear canal to measure air 
conduction and then asked patient which location sounds louder. Bone conduction better than air conduction is conductive hearing loss, and less bone conduction than air conduction is normal or sensorineural hearing loss[7].And the Weber Test uses bone conduction to determine which side of a patient's ear is damaged. The method is to put a vibrating tuning fork on the head of the patient, if the sensorineural hearing loss, then the affected side of the hearing will be reduced. In the case of conductive hearing loss, the sound heard on the affected side will be enhanced[21].

The above two methods are mostly used for rapid clinical screening due to their short time and simple test tools, but they are not accurate because they rely on the subjective feelings of patients.

\subsection{Pure tone audiometry}

Pure tone audiometry is the use of an audiometer to assess a specific frequency of hearing loss or to assess overall hearing impairment, including air conductance and bone conductance. The test starts with the patient's better ear, decreases by $10 \mathrm{~dB}$ and increases by $5 \mathrm{~dB}$ to determine the patient's hearing threshold, then tests the other ear in the same way, and finally records the data on the audiogram's horizontal axis[22].Pure tone audiometry is the gold standard of hearing assessment. However, this method also has some disadvantages. First of all, it is a subjective evaluation of hearing acuity, which makes this method inaccurate for children under 6 years old, especially some children with psychomotor disorders. Secondly, this method is also uncertain for some malingers or non-cooperative people[23].

\subsection{Auditory Steady-State Response}

Auditor-steady state response is an objective, passive, non-invasive method of hearing assessment[24]. ASSR measures hearing sensivity at specific frequencies to determine hearing thresholds for all ages. Electrophysiological audiograms can be mapped in response to activity in different parts of the brain. For example, central structures respond to slower frequency modulation, while the brainstem and peripheral auditory nerves respond to faster frequency modulation[25-27].

The advantage is that it can be used to predict true hearing thresholds in hard-to-assess patients, such as children with autism[24], and in malingering patients. ASSR also has the time-saving advantage of simultaneously measuring two ears or multiple frequencies[23]. By contrast, the disadvantage of this method is that it is greatly influenced by the individual behavioral status[28]. The second disadvantage is that when exposed to multiple stimuli simultaneously, the response range of ASSR will be reduced[29].

\subsection{Parents'/Teachers' Evaluation of Aural/Oral performance of Children}

Evaluation of Aural/Oral performance of Children is a scale used to assess the auditory performance of a hearing-impaired child in a real-world environment, which is a well-adapted valid and reliable tool for functional assessment[30]. It is divided into parent filled version (PEACH) and teacher filled version (TEACH). The questionnaire has been translated into many languages, and each version is as reliable and valid as the original one. The final score increases with age until around 20 months, the increase fades, and after 50 months, the score decreases[31].

\section{The treatments of hearing loss}

According to the different groups of people who currently treat children with hearing loss, this article will be divided into three parts, which are the treatment done by audiologists, the treatment done by auditory training software, and the adjuvant treatment done by parents of children with hearing loss.

Hearing loss includes unilateral hearing loss and bilateral hearing loss. For audiologists, surgical intervention and non-surgical intervention can be used to treat hearing loss. In the treatment of unilateral hearing loss, non-surgical methods, like FM system (a conventional choice for patients with mild to profound UHL) has a good effect for patients with mild to moderate UHL, which is more effective than conventional hearing aids. And bone-conduction hearing devices implanted surgically have significant therapeutic effects on moderate to profound UHL patients [8]. For children with mild bilateral hearing loss, proper use of amplification (FM systems and hearing aids) and environmental modification are commonly used. The main methods of environmental modification is to instruct parents to reduce the impact of background noise, such as adding carpets and curtains in the room, so as to create an acoustic-friendly environment[32]. In the treatment of bilateral hearing loss (BHL), Cochlear implantation (CI) can be used for the treatment of patients with severe to profound sensorineural hearing loss[33]. The longer a patient spends in rehabilitation training and the younger he or she is at implantation, the better his or her hearing and speech skills will recover[3]. When it comes to Auditory training (AT), person with hearing impairments actively listens to a variety of auditory stimuli in order to improve their hearing skills, and then improve their perception and discrimination of sounds[34-36]. People with mild to moderately severe hearing impairment with hearing aids or cochlear implants can be treated with AT as a subsidiary approach. As an auxiliary training method, this kind of training is helpful to improve the communication ability and quality of life of patients[36] [37]

With the development of current science and technology, many auditory training software based on mobile phone or computer and other electronic products also play an important role in hearing rehabilitation. This kind of software can make listening training independent of time factors and make the data of training stored in the cloud accessible to both patients and audiologists[10]. Listening training based on virtual reality or immersive virtual reality is a suitable auditory training tool for 
children with cochlear implant or hearing aid. Such methods use special devices such as Head-Mounted Displays (HMD) to train children in auditory skills such as sound detection, auditory discrimination, auditory recognition, and auditory comprehension[38]. The theory is based on Erber's paradigm of stages of auditory development[39]. This kind of software integrates interesting games with real scenes, which greatly stimulates the training motivation of children and makes listening training more acceptable to children and speeds up the process of hearing recovery process[40]. And children's therapists can also obtain accurate training data through the software.

With regard to family involvement, the use of the Group of Family Involvement (GrAF) at the initial stages of the intervention is currently the common approach. Audiologists discuss the child's hearing potential and intervention options with parents through regular meetings [9], and teach the use of hearing AIDS and daily care methods[41, 42]. Many studies have shown that early intervention is the key to hearing rehabilitation in children[2]. Hence, the first advantage is that parents are the ones who have the most contact with their children and the earliest, and are able to carry out early intervention well. In addition, after training, parents can ensure the duration and effectiveness of hearing aid use in children to a large extent, and the system and continuous use of hearing aid are important for children's hearing and language function. Furthermore, the paper points out that parents' motivational strategies and expectations can influence family outcomes in hearing rehabilitation[9]. Different from normal children, the attention of children with hearing impairment will be affected by the direction of their parents' gaze and hand movements. Therefore, it is very important for parents to take the initiative and correct accompanying training in daily social interactions[43]. Parents of hearing impaired children can also evaluate themselves through the Scale of Parental Involvement and self-efficacy (SPISE)[44].

\section{Conclusion}

The causes of hearing loss can be divided into hereditary causes and acquired causes. The hereditary causes are mainly related to the mutation of genes, while the acquired causes are related to the impairment of the outer, middle and inner ears. Assessment methods for hearing impairment include The Rinne Test and The Weber Test, Pure tone audiometry, sound-steady State Response and PEACH/TEACH. At present, the commonly used methods of hearing treatment include surgical intervention and non-surgical intervention conducted by audiologists. Methods carried out by hearing training software combined with virtual reality game and methods involving family early involvement intervention. It is hoped that future research can further explore the causes of hearing impairment, and design a software platform that can promote closer interaction between audiologists and parents while providing simple listening training functions.

\section{References}

1. Graydon, K., et al., Global burden of hearing impairment and ear disease. The Journal of Laryngology \& Otology, 2018. 133(1): p. 18-25.

2. Bess, F.H. and A. Tharpe, Performance and management of children with unilateral sensorineural hearing loss. Scandinavian audiology, 1988.

3. Baser, B., M. Patidar, and P. Surana, A Study to Determine Various Factors Influencing Auditory Outcomes in Paediatric Cochlear Implantation. Indian Journal of Otolaryngology and Head \& Neck Surgery, 2020. 72(4): p. 453-456.

4. Lin, K.-J. and Y.-C. Hung, Monitoring children with hearing fluctuation. The Hearing Journal, 2017. 70(10): p. 14-16.

5. Anthwal, N. and H. Thompson, The development of the mammalian outer and middle ear. Journal of anatomy, 2016. 228(2): p. 217-232.

6. Trott, K., et al., Auditory Rehabilitation in Children with Cerebral Palsy. Cerebral Palsy, 2020: p. 811-818.

7. Isaacson, J. and N.M. Vora, Differential diagnosis and treatment of hearing loss. American family physician, 2003. 68(6): p. 1125-1132.

8. Appachi, S., et al., Auditory outcomes with hearing rehabilitation in children with unilateral hearing loss: a systematic review. Otolaryngology-Head and Neck Surgery, 2017. 157(4): p. 565-571.

9. Miguel, J.H.d.S. and B.C.d.A.C. Novaes, Hearing rehabilitation in children: adhesion to treatment and use of hearing aids. Audiology-Communication Research, 2013. 18(3): p. 171-178.

10. Clark, J.L. and D.W. Swanepoel, Technology for hearing loss-as we know it, and as we dream it. Disability and Rehabilitation: Assistive Technology, 2014. 9(5): p. 408-413.

11. Borck, G., et al., Loss-of-function mutations of ILDR1 cause autosomal-recessive hearing impairment DFNB42. The American Journal of Human Genetics, 2011. 88(2): p. 127-137.

12. Santos-Cortez, R.L.P., et al., Mutations in KARS, encoding lysyl-tRNA synthetase, cause autosomal-recessive nonsyndromic hearing impairment DFNB89. The American Journal of Human Genetics, 2013. 93(1): p. 132-140.

13. Vona, B., et al., DFNB16 is a frequent cause of congenital hearing impairment: implementation of STRC mutation analysis in routine diagnostics. Clinical genetics, 2015. 87(1): p. 49-55.

14. Clark, W.B., et al., Microbiology of otitis externa. Otolaryngology-Head and Neck Surgery, 1997. 116(1): p. 23-25.

15. Mulwafu, W., H. Kuper, and R. Ensink, Prevalence and causes of hearing impairment in Africa. Tropical medicine \& international health, 2016. 21(2): p. 158-165. 
16. Gravel, J.S. and I.F. Wallace, Effects of otitis media with effusion on hearing in the first 3 years of life. Journal of Speech, Language, and Hearing Research, 2000. 43(3): p. 631-644.

17. Graydon, K., et al., Global burden of hearing impairment and ear disease. The Journal of Laryngology \& Otology, 2019. 133(1): p. 18-25.

18. Ghafari, N., et al., The occurrence of auditory dysfunction in children with TB receiving ototoxic medication at a TB hospital in South Africa. International journal of pediatric otorhinolaryngology, 2015. 79(7): p. 1101-1105.

19. Lanvers-Kaminsky, C., et al., Drug-induced ototoxicity: mechanisms, pharmacogenetics, and protective strategies. Clinical pharmacology \& therapeutics, 2017. 101(4): p. 491-500.

20. Organization, W.H., Childhood hearing loss: strategies for prevention and care. 2016.

21. Phillips, M., et al., Analysis of results from the Kraus-Weber test of minimum muscular fitness in children. Research Quarterly. American Association for Health, Physical Education and Recreation, 1955. 26(3): p. 314-323.

22. Walker, J.J., et al., Audiometry screening and interpretation. American family physician, 2013. 87(1): p. 41-47.

23. Swami, H. and S. Kumar, Comparison of frequency-Specific hearing thresholds between pure-tone audiometry and auditory steady-state response. Indian Journal of Otology, 2019. 25(2): p. 59.

24. Ono, Y., et al., Auditory steady-state response at 20 $\mathrm{Hz}$ and $40 \mathrm{~Hz}$ in young typically developing children and children with autism spectrum disorder. Psychiatry and Clinical Neurosciences, 2020. 74(6): p. 354-361.

25. Sininger, Y.S. and C. Abdala, Hearing threshold as measured by auditory brain stem response in human neonates. Ear and hearing, 1996. 17(5): p. 395-401.

26. Valdes, J.L., et al., Comparison of statistical indicators for the automatic detection of $80 \mathrm{~Hz}$ auditory steady state responses. Ear and hearing, 1997. 18(5): p. 420-429.

27. John, M.S., et al., Multiple auditory steady-state responses (MASTER): stimulus and recording parameters. Audiology, 1998. 37(2): p. 59-82.

28. Ahn, J.H., et al., Comparing pure-tone audiometry and auditory steady state response for the measurement of hearing loss. Otolaryngology-Head and Neck Surgery, 2007. 136(6): p. 966-971.

29. Stevens, J., State of the art neonatal hearing screening with auditory brainstem response. Scandinavian Audiology, 2001. 30(1): p. 10-12.

30. Fatahi, F., et al., Development of Persian version of teachers' evaluation of aural/oral performance of children scale. Auditory and Vestibular Research, 2020 .
31. Brännström, K.J., et al., Clinical note: Validation of the Swedish version of the Parents' Evaluation of Aural/Oral Performance of Children (PEACH) Rating Scale for normal hearing infants and children. Hearing, Balance and Communication, 2014. 12(2): p. 88-93.

32. Holstrum, W.J., et al., Early intervention for children with unilateral and mild bilateral degrees of hearing loss. Trends in Amplification, 2008. 12(1): p. 35-41.

33. Gaurav, V., S. Sharma, and S. Singh, Effects of Age at Cochlear Implantation on Auditory Outcomes in Cochlear Implant Recipient Children. Indian Journal of Otolaryngology and Head \& Neck Surgery, 2020. 72(1): p. 79-85.

34. Henshaw, H. and M.A. Ferguson, Efficacy of individual computer-based auditory training for people with hearing loss: a systematic review of the evidence. PloS one, 2013. 8(5): p. e62836.

35. Olson, A.D. Options for auditory training for adults with hearing loss. in Seminars in hearing. 2015. Thieme Medical Publishers.

36. Stropahl, M., J. Besser, and S. Launer, Auditory training supports auditory rehabilitation: A state-of-the-art review. Ear and hearing, 2020. 41(4): p. 697-704.

37. Boothroyd, A., Adapting to changed hearing: the potential role of formal training. Journal of the American Academy of Audiology, 2010. 21(9): p. 601-611.

38. Hatzigiannakoglou, P.D. and A. Okalidou, Development of an Auditory Rehabilitation Tool for children with Cochlear Implants through a Mobile-Based VR and AR serious game. International Journal of Online and Biomedical Engineering (iJOE), 2019. 15(02): p. 81-90.

39. Hack, Z.C. and N.P. Erber, Auditory, visual, and auditory-visual perception of vowels by hearing-impaired children. Journal of Speech, Language, and Hearing Research, 1982. 25(1): p. 100-107.

40. Sveistrup, H., Motor rehabilitation using virtual reality. Journal of neuroengineering and rehabilitation, 2004. 1(1): p. 10.

41. Sjoblad, S., et al., Parents' reactions and recommendations after diagnosis and hearing aid fitting. American Journal of Audiology, 2001.

42. Brazorotto, J., A terapia fonoaudiológica da criança surda. Bevilaqua MC, Moret ALM. Deficiência auditiva: conversando com familiares e profissionais de saúde. São José dos Campos: Pulso, 2005: p. 203-25.

43. Chen, C.h., et al., What leads to coordinated attention in parent-toddler interactions? Children's hearing status matters. Developmental Science, 2020. 23(3): p. e12919.

44. Moeller, M.P., Early intervention and language development in children who are deaf and hard of hearing. Pediatrics, 2000. 106(3): p. e43-e43. 